\title{
EELS Spectrum Imaging: The Next Steps
}

\author{
G. Kothleitner, B. Schaffer, W. Grogger and F. Hofer
}

Research Institute f. Electron Microscopy (FELMI), Graz University of Technology

Steyrergasse 17, A-8010 Graz, Austria

With the event of energy-filtering transmission electron microscopes, in combination with fast two-dimensional EELS detection systems, more powerful acquisition and processing software and the enormous increase in computing power, there has been growing interest in exploiting the potential imaging capabilities of electron energy-loss spectroscopy for the comprehensive compositional and electronic characterization of modern materials. Especially the EELS spectrum imaging technique by now has gained widespread acceptance as a tool that not only provides better mechanisms to cross-check the data quality but also allows for increased sophistication in processing the data compared to conventional 3-window elemental mapping or EELS point analysis.

The idea of EELS spectrum imaging is to collect an almost complete data set in the spatial and energy-loss dimensions. This can be achieved by either recording an entire energy-loss spectrum from each pixel in an image (STEM SI) [1] or by recording the spatial distribution of the intensity at each energy-loss (EFTEM SI) [2]. Various forms of spectrum image data processing like thickness deconvolution [3], Kramers-Kronig analysis, MLS-fitting for quantitative compositional image analysis [4,5] or automated elemental occurrence mapping [5] have already been successfully demonstrated. However, depending on the acquisition mode, most spectrum imaging applications so far were either limited in energy resolution and/or field of view. With the latest generation of monochromated TEMs, reaching energy resolutions of as low as $0.1-0.3 \mathrm{eV}$ [6,7], spectrum imaging has reached the next challenge level.

For applications in the low-loss region, the EFTEM spectrum imaging approach has several advantages, which for instance include the fact that the imaged areas can be larger and the exposure times can still be kept relatively low even when the slit widths used are very small $(\sim 0.2 \mathrm{eV})$ or the monochromator is switched on. Although current imaging filters are corrected up to $3^{\text {rd }}$ and partly $4^{\text {th }}$ order [8], higher-order spectral aberrations (non-isochromaticity) can become visible under these conditions, preventing narrow low-loss features to be mapped uniformly. Provided there was no sample drift between the image slices, the contribution of energy warping to the individual image plane intensities could be determined and corrected. However, in the case of drift, since each image plane has its individual displacement vector, which shifts the respective non-isochromatic surface correspondingly, a combined spectral aberration and spatial drift correction is not straightforward but always has to be carried out simultaneously, in order to avoid the introduction of additional artifacts. In this paper we will give an overview of recent EELS spectrum imaging applications, and present a new artifact correction scheme to comply with the higher resolution requirements of present-day applications.

References

[1] C. Jeanguillaume and C. Colliex Ultramicroscopy 28 (1989) 252.

[2] J.L. Lavergne et al Microsc. Microanal. Microstruct. 3 (1992) 517.

[3] P.J. Thomas and P.A. Midgley Ultramicroscopy 88 (2001) 187.

[4] M. Tencé et al Ultramicroscopy 58 (1995) 42.

[5] G. Kothleitner and F. Hofer Ultramicroscopy 96 (2003) 491. 
[6] G. Kothleitner and F. Hofer Micron 34 (2003) 211.

[7] S. Lazar et al Ultramicroscopy 96 (3-4) (2003) 535.

[8] H. A. Brink et al Ultramicroscopy 96 (3-4) (2003) 367.

[9] Special thanks to Dr. A. Trampert (P. Drude Institute Berlin, Germany) for providing the AlN/GaN sample and to the SFB "Electroactive Materials" for financial support.

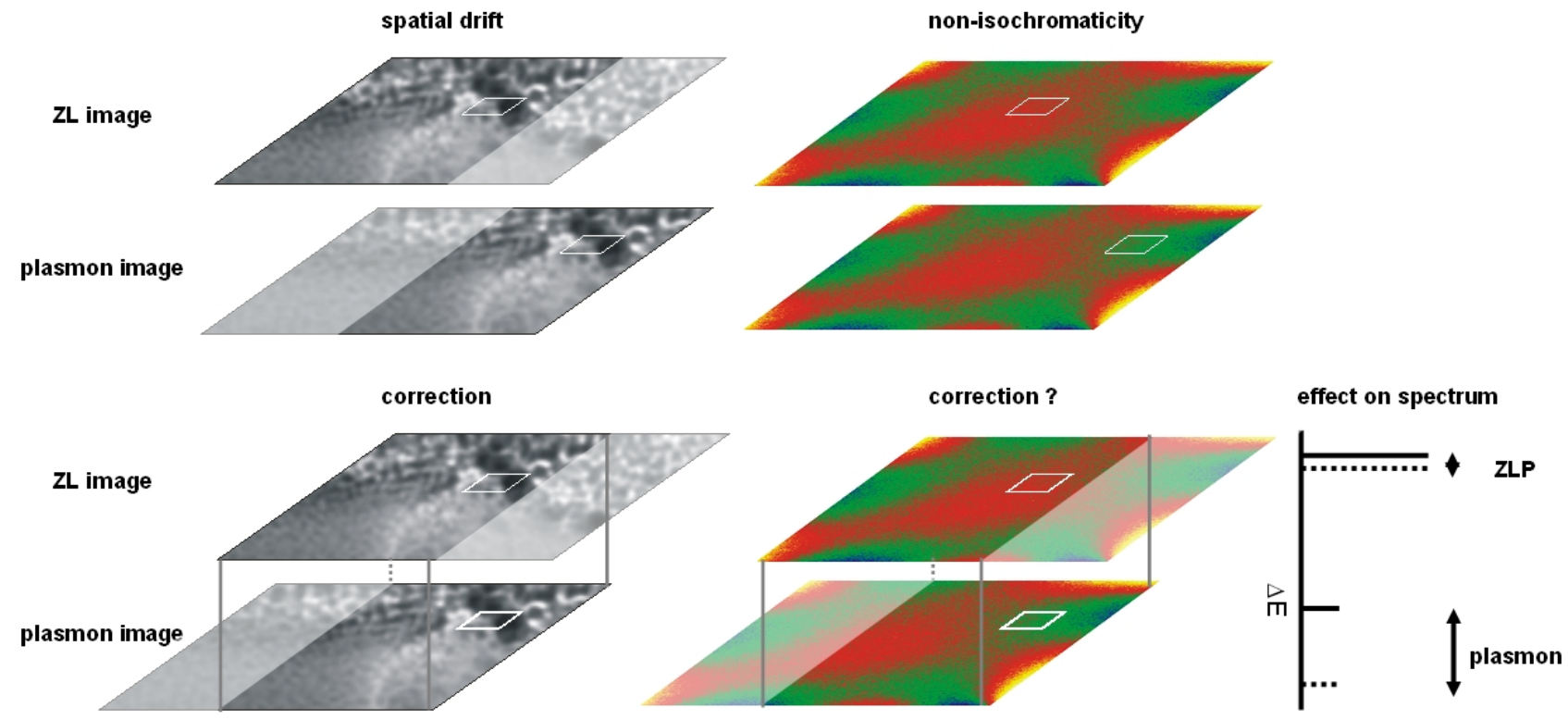

FIG. 1.Graphics, showing the implications of simultaneous spatial drift and spectral aberration correction.
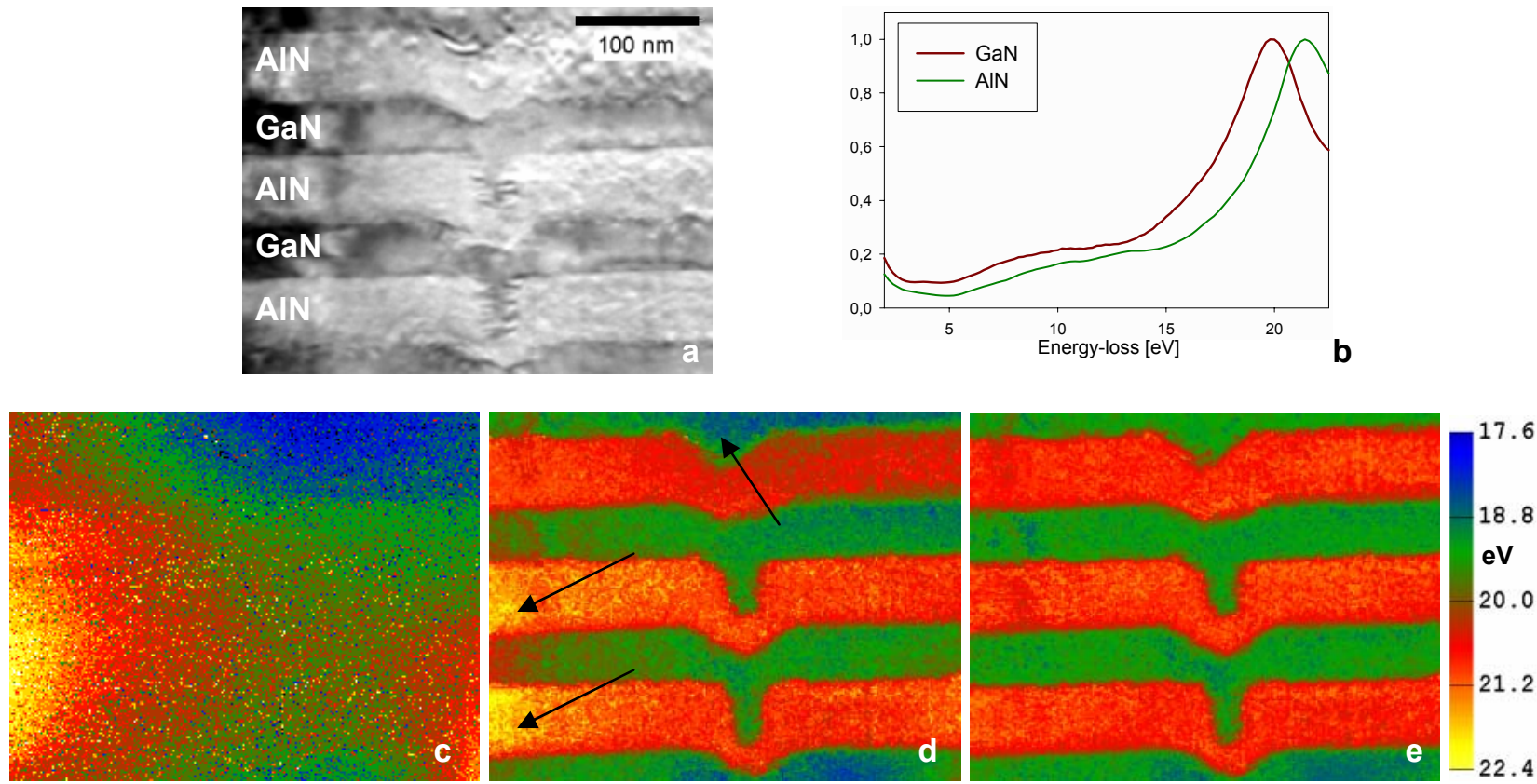

FIG. 2. a) ZL-filtered image of a layered AIN/GaN stack and b.) corresponding plasmon spectra. c.) spectral aberrations together with d.) incorrectly and e) correctly calculated plasmon shift map. 